\title{
The Design of the IIR Differintegrator and its Application in Edge Detection
}

\author{
Madhu Jain*, Maneesha Gupta**, and N. K. Jain***
}

\begin{abstract}
New IIR digital differintegrators (differentiator and integrator) with very minor absolute relative errors are presented in this paper. The digital integrator is designed by interpolating some of the existing integrators. The optimum value of the interpolation ratio is obtained through linear programming optimization. Subsequently, by modifying the transfer function of the proposed integrator appropriately, new digital differentiator is obtained. Simulation results demonstrate that the proposed differintegrator are a more accurate approximation of ideal ones, than the existing differintegrators. Furthermore, the proposed differentiator has been tested in an image processing application. Edges characterize boundaries and are, therefore, a problem of fundamental importance in image processing. For comparison purpose Prewitt, Sobel, Roberts, Canny, Laplacian of Gaussian (LOG), Zerocross operators were used and their results are displayed. The results of edge detection by some of the existing differentiators are also provided. The simulation results have shown the superiority of the proposed approach over existing ones.
\end{abstract}

Keywords-Digital integrator, digital differentiator, edge detection and image processing

\section{INTRODUCTION}

Digital differintegrators (differentiators and integrators) are important components in almost all engineering disciplines, including controls, communications, biomedical, and image processing applications. The frequency response of an ideal digital differintegrator is $H_{I}(\omega)=(j \omega)^{ \pm 1}$, Where, $j=$ $\sqrt{ }-1$, ' +1 ' is for the differentiator, ' -1 ' is for the integrator, and $\omega$ is the angular frequency in radians.

Due to their large number of applications, considerable interest in the design of suitable digital differintegrators has encouraged the development of various techniques. The objective is to design these differintegrators so that they meet the given requirements with sufficient accuracy. Various approximations of ideal differintegrators have been reported in the literature [1-9]. Al-Alaoui [1] has proposed the interpolation method to design a digital integrator. Schneider et al. [2] have also proposed a digital integrator by modifying the Newton-Cotes integration rule. Papamarkos and Chamzas [3] used the linear programming optimization method in designing digital integrators. Ngo [4] used the closed form of the Newton-Cotes integration rule to design a digital integrator. Al-Alaoui [5] used fractional delay in their design of digital integrators. A family of digital integrators and differentiators has also been proposed by Al-Alaoui [6] by using interpolation and a

\footnotetext{
Manuscript received April 11, 2013 ; first revision November 21, 2013; accepted February 13, 2014.

Corresponding Author : Madhu Jain (ermadhu2003@gmail.com)

* Department of Electronics and Communication Engineering, Jaypee Institute of Information Technology (ermadhu2003@gmail.com)

** Netaji Subhas Institute of Technology, Department of Electronics and Communication Engineering (maneeshapub@gmail.com)

*** Indian Institute of tecnology (IIT) Delhi, Instrument design development centre (ntnjain@gmail.com)
} 
simulated annealing optimization method. Gupta-Jain-Kumar (GJK) [7-9] have also proposed digital integrators by using the interpolation method. These approximations only meet the magnitude or linear phase requirement, but do not meet both simultaneously. A differintegrator should have a linear phase response over the wideband frequency range. Otherwise, it would introduce phase distortion in the system in which it is incorporated. The design problem of a linear phase differintegrator is a challenging task. Therefore, there is a strong motivation to design linear phase differintegrators with magnitude characteristics that are close to the ideal differintegrator. In this paper, an attempt has been made to fulfill the gap between the desired and existing responses. Here, five new approximations of an ideal digital integrator arepresented. The approximations are based on an interpolation of some already known digital integrators. So far, in the interpolation method, the interpolation ratio has been obtained over a narrow range of values. While, in this paper, the interpolation ratio is obtained through linear programming that extends over a wider range of 999 values to ensure that the result is optimized. Out of five, the integrator with superior frequency response is chosen. Afterwards, by modifying the transfer function of the proposed integratorcongruously, the digital differentiator is also obtained. The simulation result shows that the proposed differintegrator outperforms all of the existing ones over the entire Nyquist frequency range, in terms of both magnitude and phase response. That makes them useful in various kinds of practical applications. To demonstrate the practical ability of the proposed differentiator, it is applied for edge detection in image processing. It is well known that edge detection conserves the important structural properties, while dislodging useless information in an image that makes it the nucleus of an investigation in several image processing applications. Distinct edge detection algorithms have been developed in the process of finding the perfect edge detector [10-12]. We designed the algorithm for edge detection on the software MATLAB 7 and compared the results with existing methods. All the analysis figures in this paper were obtained using MATLAB. The sampling period of the filter ' $T$ ' is assumed as 1second in the frequency plots and the Nyquist frequency is $\pi$ radians. This paper is organized as follows: Section 2 gives the design of the proposed digital integrator and compares it with the existing integrators. Section 3 gives the design of the proposed digital differentiators and compares it with the existing differentiators. The application of the proposed differentiator for edge detection is discussed in Section 4. The conclusions are given in Section 5.

\section{Design of the Proposed Digital Integrator and its Comparison WITH THE EXISTING INTEGRATORS}

\subsection{Design of the Proposed Integrator}

In this section, some of the existing integrators are linearly interpolated to develop new integrators that could better approximate the ideal integrator. The concept of linear interpolation is based on thepresumptionthat mixing two good integrators can result in a better integrator [1].

In this technique, a set of two integrators is chosen from the existing integrators. The coefficients of these two integrators are linearly interpolated to develop a new integrator, which could better approximate the ideal integrator. The interpolation ratio is a user- specified weight that balances the impact of the selected integrators.

The new integrator, $H_{N}(z)$, can be mathematically stated as: 


$$
\mathrm{H}_{\mathrm{N}}(\mathrm{z})=\alpha \mathrm{H}_{1}(\mathrm{z})+(1-\alpha) \mathrm{H}_{2}(\mathrm{z})
$$

Where, $H_{N}(z)$ is the transfer function of new integrator, $H_{1}(z)$ and $H_{2}(z)$ are the transfer functions of the existing integrators. The factor $\alpha,(0<\alpha<1)$ determines the fraction ofcontribution of each integrator in the new integrator.

Existing digital integrators with their transfer functions are mentioned below. They are as follows:

Trapezoidal integrator [1]

$$
\mathrm{H}_{\mathrm{T}}(\mathrm{z})=\frac{0.5 \mathrm{~T}(\mathrm{z}+1)}{(\mathrm{z}-1)}
$$

Rectangular integrator [1]

$$
\mathrm{H}_{\mathrm{R}}(\mathrm{z})=\frac{\mathrm{T}}{(\mathrm{z}-1)}
$$

Schneider-Kaneshige-Groutage (SKG) integrator [2]

$$
\mathrm{H}_{\mathrm{SKG}}(\mathrm{z})=\frac{\mathrm{T}\left(9 \mathrm{z}^{3}+19 z^{2}-5 \mathrm{z}+1\right)}{24 \mathrm{z}^{2}(\mathrm{z}-1)}
$$

Ngo integrator [4]

$$
\mathrm{H}_{\mathrm{Ngo}}(\mathrm{z})=\frac{\mathrm{T}(\mathrm{z}+2.3658)\left(\mathrm{z}^{2}-0.2605 \mathrm{z}+0.047\right)}{2.7925 \mathrm{z}^{2}(\mathrm{z}-1)}
$$

Al-Alaoui optimized 3-Segment integrator [6]

$$
\mathrm{H}_{\mathrm{AO} 3}(\mathrm{z})=\frac{\mathrm{T}\left(0.06471 \mathrm{z}^{3}+0.1617 \mathrm{z}^{2}+0.9625 \mathrm{z}+0.8315\right)}{\left(\mathrm{z}^{3}-0.2389 \mathrm{z}^{2}-0.4987 \mathrm{z}-0.2619\right)}
$$

Al-Alaoui optimized 4-Segment integrator [6]

$$
\mathrm{H}_{\mathrm{AO} 4}(\mathrm{z})=\frac{\mathrm{T}\left(0.5678 \mathrm{z}^{4}+1.013 \mathrm{z}^{3}+0.5629 \mathrm{z}^{2}+0.5406 \mathrm{z}+0.1519\right)}{\left(\mathrm{z}^{4}-0.2097 \mathrm{z}^{3}-0.2106 \mathrm{z}^{2}-0.114 \mathrm{z}-0.4636\right)}
$$

Gupta-Jain-Kumar 1 (GJK1) integrator [8]

$$
\mathrm{H}_{\mathrm{GJK} 1}(\mathrm{z})=\frac{0.34 \mathrm{~T}(\mathrm{z}+2.541)\left(\mathrm{z}^{2}-0.2081 \mathrm{z}+0.03858\right)}{\mathrm{z}^{2}(\mathrm{z}-1)}
$$

Gupta-Jain-Kumar 2 (GJK2) integrator [9]

$$
\mathrm{H}_{\mathrm{GJK} 2}(\mathrm{z})=\frac{0.329 \mathrm{~T}(\mathrm{z}+2.663)\left(\mathrm{z}^{2}-0.2079 \mathrm{z}+0.03864\right)}{\mathrm{z}^{2}(\mathrm{z}-1)}
$$


To find the optimum value of the interpolation ratio $(\alpha)$, a linear programming algorithm has been designed. Here, the percentage absolute relative error (PARE) of the new integrator with respect to the ideal one is taken as an objective function. It is defined as:

$$
\operatorname{PARE}(\omega)=\frac{|| \mathrm{H}_{\mathrm{I}}(\omega)|-| \mathrm{H}_{\mathrm{N}}\left(\mathrm{e}^{\mathrm{j} \omega \mathrm{T}}\right) \|}{\left|\mathrm{H}_{\mathrm{I}}(\omega)\right|} * 100
$$

Where, $H_{I}(\omega)=1 /(j \omega)$ and $H_{N}\left(e^{j \omega T}\right)$ is calculated by replacing $z$ by $e^{j \omega T}$ in Eq. (1). The objective of the algorithm is to minimize the objective function and can be interpreted as:

$$
\begin{aligned}
& \operatorname{minimize} \mu \\
& \text { subject to }|\operatorname{PARE}(\omega)| \leq \mu
\end{aligned}
$$

Where, variable $\mu$ defines the value of the maximum PARE. Here, the range of frequency $(\omega)$ is taken as $\omega \in[0, \pi]$ radians and the range of the interpolation ratio is taken as $\alpha \in[0.001$, 0.999]. The flowchart of the proposed linear programming algorithm is shown in Figure 1.

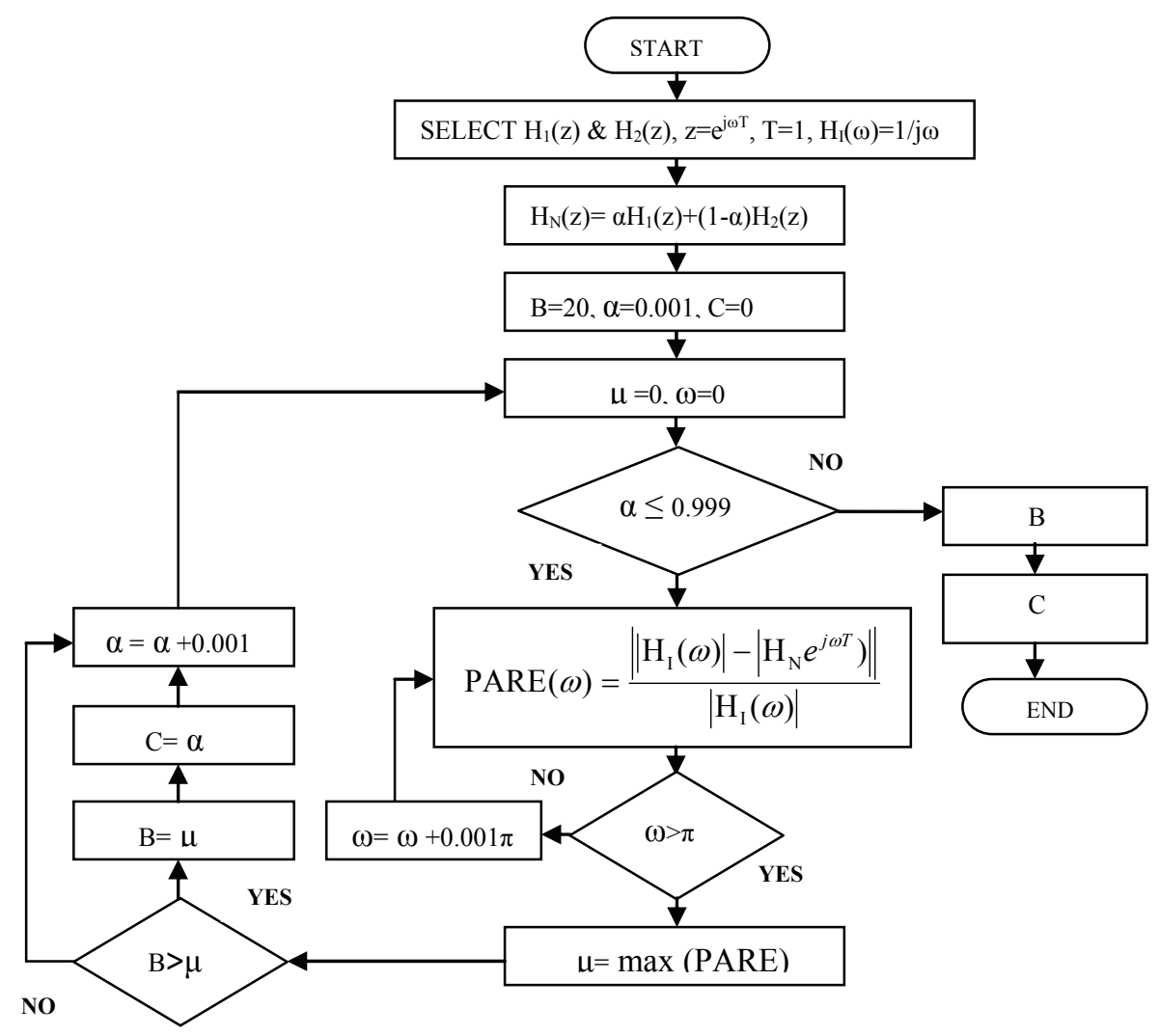

Fig. 1. Flowchart of the proposed algorithm 
First of all, two integer order integrators are selected from among the existing ones. Here, variables $C$ and $B$ are used to define the optimum interpolation ratio and optimum maximum $P A R E$, respectively. The initial values are selected as $C=0$ and $B=20$. The selected integrators are mixed for $\alpha=0.001$ and PARE is evaluated at each frequency in duration of $0.001 \pi$. After this $\mu$ is calculated and compared with $B$. In the case when $B$ is greater than $\mu$, the value of $\mu$ and $\alpha$ is copied in $B$ and $C$, respectively. Furthermore, $\alpha$ is increased by 0.001 and by applying the above-mentioned steps the value of $B$ and $C$ is calculated. Subsequently, this process is repeated until the maximum value of $\alpha(0.999)$ is reached. In the end of the algorithm, the final values of $B$ and $C$ are obtained. Five new integrators are obtained by applying the proposed algorithm on some of the existing integrators.

These are as follows:

(a) Interpolation of $H_{N g o}(z)$ and $H_{T}(z)$ :

The new transfer function is:

$$
\mathrm{H}_{\mathrm{N} 1}(\mathrm{z})=\alpha \mathrm{H}_{\mathrm{Ngo}}(\mathrm{z})+(1-\alpha) \mathrm{H}_{\mathrm{T}}(\mathrm{z})
$$

By implementing the proposed algorithm the optimum value of $\alpha$ and maximum PARE is obtained as 0.91 and 12.05 , respectively. By substituting the value of $\alpha, H_{N g o}(z)$ and $H_{T}(z)$ in Eq. (11) the achieved transfer function is:

$$
\mathrm{H}_{\mathrm{N} 1}(\mathrm{z})=\frac{0.37087 \mathrm{~T}(\mathrm{z}+2.217)\left(\mathrm{z}^{2}-0.2455 \mathrm{z}+0.04407\right)}{\mathrm{z}^{2}(\mathrm{z}-1)}
$$

(b) Interpolation of $H_{S K G}(z)$ and $H_{R}(z)$ :

The new transfer function is:

$$
\mathrm{H}_{\mathrm{N} 2}(\mathrm{z})=\alpha \mathrm{H}_{\mathrm{SKG}}(\mathrm{z})+(1-\alpha) \mathrm{H}_{\mathrm{R}}(\mathrm{z})
$$

By implementing the proposed algorithm, the optimum value of $\alpha$ and the maximum PARE is obtained as 0.91 and 9.1221, respectively. By substituting the optimum value of $\alpha, H_{S K G}(z)$ and $H_{R}(z)$ in Eq. (13) the resulting transfer function is:

$$
\mathrm{H}_{\mathrm{N} 2}(\mathrm{z})=\frac{0.34312 \mathrm{~T}(\mathrm{z}+2.59)\left(\mathrm{z}^{2}-0.2311 \mathrm{z}+0.0429\right)}{\mathrm{z}^{2}(\mathrm{z}-1)}
$$

(c) Interpolation of $H_{S K G}(z)$ and $H_{N g o}(z)$ :

The new transfer function is:

$$
\mathrm{H}_{\mathrm{N} 3}(\mathrm{z})=\alpha \mathrm{H}_{\mathrm{SKG}}(\mathrm{z})+(1-\alpha) \mathrm{H}_{\mathrm{Ngo}}(\mathrm{z})
$$

By implementing the proposed algorithm, the optimum value of $\alpha$ and the maximum PARE is obtained as 0.94 and 6.1558, respectively. By substituting the optimum value of $\alpha, H_{S K G}(z)$ and $H_{N g o}(z)$ in Eq. (15) the resulting transfer function is: 


$$
\mathrm{H}_{\mathrm{N} 3}(\mathrm{z})=\frac{0.37399 \mathrm{~T}(\mathrm{z}+2.366)\left(\mathrm{z}^{2}-0.2547 \mathrm{z}+0.04697\right)}{\mathrm{z}^{2}(\mathrm{z}-1)}
$$

(d) Interpolation of $H_{S K G}(z)$ and $H_{T}(z)$ :

The new transfer function is:

$$
\mathrm{H}_{\mathrm{N} 4}(\mathrm{z})=\alpha \mathrm{H}_{\mathrm{SKG}}(\mathrm{z})+(1-\alpha) \mathrm{H}_{\mathrm{T}}(\mathrm{z})
$$

By implementing the proposed algorithm, the optimum value of $\alpha$ and the maximum PARE is obtained as 0.95 and 6.4814, respectively. By substituting the optimum value of $\alpha, H_{S K G}(z)$ and $H_{T}(z)$ in eq. (17) the realized transfer function is:

$$
\mathrm{H}_{\mathrm{N} 4}(\mathrm{z})=\frac{0.38125 \mathrm{~T}(\mathrm{z}+2.285)\left(\mathrm{z}^{2}-0.247 \mathrm{z}+0.04543\right)}{\mathrm{z}^{2}(\mathrm{z}-1)}
$$

(e) Interpolation of $H_{S K G}(z)$ and $H_{A O 3}(z)$ :

The new transfer function is:

$$
\mathrm{H}_{\mathrm{N} 5}(\mathrm{z})=\alpha \mathrm{H}_{\mathrm{SKG}}(\mathrm{z})+(1-\alpha) \mathrm{H}_{\mathrm{AO} 3}(\mathrm{z})
$$

By implementing the proposed algorithm, the optimum value of $\alpha$ and the maximum PARE is obtained as 0.975 and 2.4, respectively. By substituting the optimum value of $\alpha, H_{S K G}(z)$ and $H_{A O 3}(z)$ in eq. (19) the obtained transfer function is:

$$
\mathrm{H}_{\mathrm{N} 5}(\mathrm{z})=\frac{0.3540 \mathrm{~T}(\mathrm{z}+2.4582)\left(\mathrm{z}^{2}-0.2190 \mathrm{z}+0.0497\right)\left(\mathrm{z}^{2}+0.7611 \mathrm{z}+0.268\right)}{\mathrm{z}^{2}(\mathrm{z}-1)\left(\mathrm{z}^{2}+0.7609 \mathrm{z}+0.262\right)}
$$

Here, the polynomials in the numerator and denominator are $\left(z^{2}+0.7611 z+0.268\right)$ and $\left(z^{2}+0.7609 z+0.262\right)$, respectively. They have coefficients that are very close to each other and their cancellations do not seriously affect the response. Therefore, the reduced transfer function is given by:

$$
\mathrm{H}_{\mathrm{N} 5}(\mathrm{z})=\frac{0.3540 \mathrm{~T}(\mathrm{z}+2.4582)\left(\mathrm{z}^{2}-0.2190 \mathrm{z}+0.0497\right)}{\mathrm{z}^{2}(\mathrm{z}-1)}
$$

The PARE response of the designed integrators (defined in Eqs. 12, 14, 16, 18, and 20b) is calculated with the help of Eq. (10) and are shown in Figure 2.

It can be observed that integrator $H_{N S}(z)$ has $P A R E \leq 2.4$ over the entire Nyquist frequency range and outperforms the other designed integrators. Thus, it is known as the proposed integrator throughout this paper. 


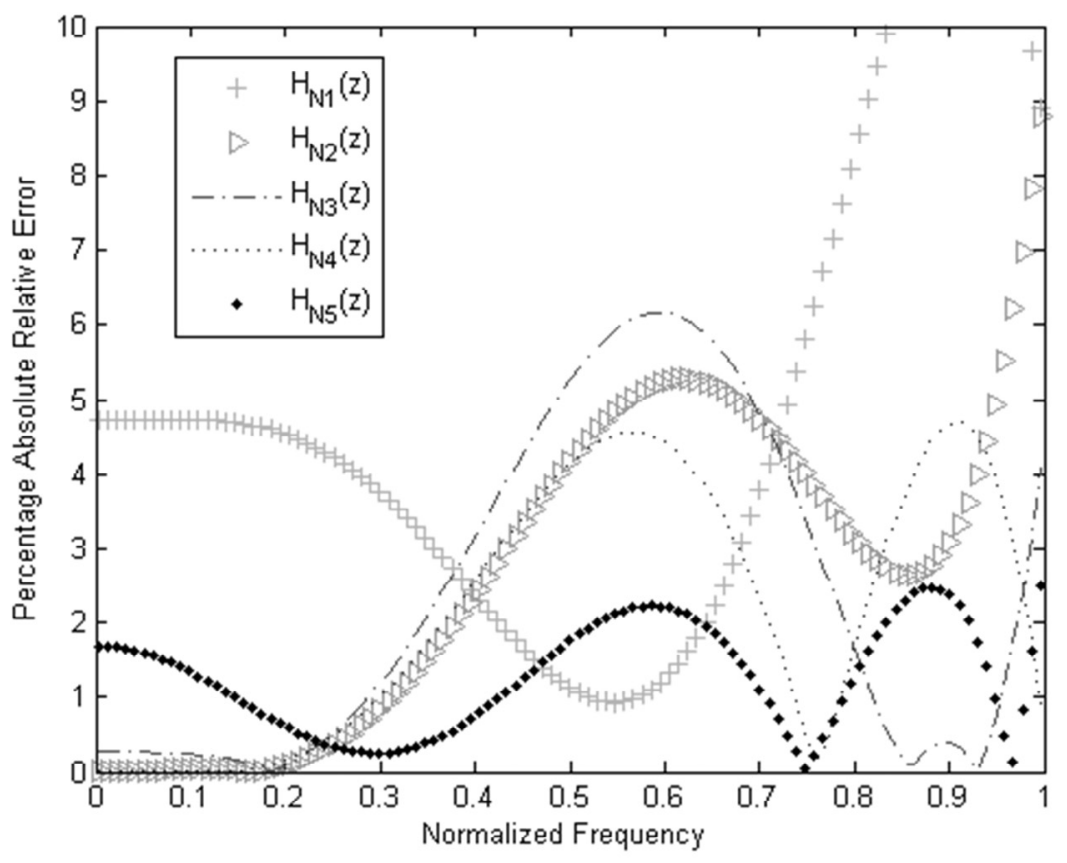

Fig. 2. PARE response of the designed integrators: $H_{N 1}(z), H_{N 2}(z), H_{N 3}(z), H_{N 4}(z)$, and $H_{N 5}(z)$

\subsection{Comparison of theProposed Integrator with the Existing Integrators}

To define the efficiency of the proposed integrator, its comparison with the existing integrators (the transfer functions mentioned in Section 2.1) is shown in respect of their frequency response. Frequency response analysis is performed in terms of PARE and phase response in Figure 3 and 4, respectively. The PARE response of the existing integrators is calculated with the help of Eq. (10) and their maximum PARE over the entire Nyquist frequency range is shown in Table 1.

It is evident from the above results that the proposed integrator $H_{N S}(z)$ is the closest approximation of the ideal integrator, as compared to existing ones over the entire Nyquist frequency range: $0 \leq \omega \leq \pi$ radians.

Measuring deviation from the linear phase is a way to define non-linearity in the phase response. This can be accomplished by calculating the difference between the proposed phase andthe ideal one at each frequency. The maximum phase deviation of the proposed integratorfrom the ideal linear phase response is $33.4^{\circ}$ (which occurs at $\omega=0.57 \pi$ radians). While the maximum phase deviation of the existing integrators $S K G$ [2], Al-Alaoui optimized 4-Segment [6], Gupta-JainKumar 1 [8], and Gupta-Jain-Kumar 2 [9] from the ideal linear phase response is $35.5^{\circ}$ (which occurs at $\omega=0.61 \pi$ radians), $77.80^{\circ}$ (which occurs at $\omega=0.80 \pi$ radians), $35.3^{\circ}$ (which occurs at $\omega=0.59 \pi$ radians), and $34.2^{\circ}$ (which occurs at $\omega=0.58 \pi$ radians), respectively.

These results (Figures 3 and 4 and Table 1) confirm that the proposed integrator $H_{N 5}(z)$ outperforms all of the existing integrators in magnitude and phase response over the entire Nyquist frequency range. 


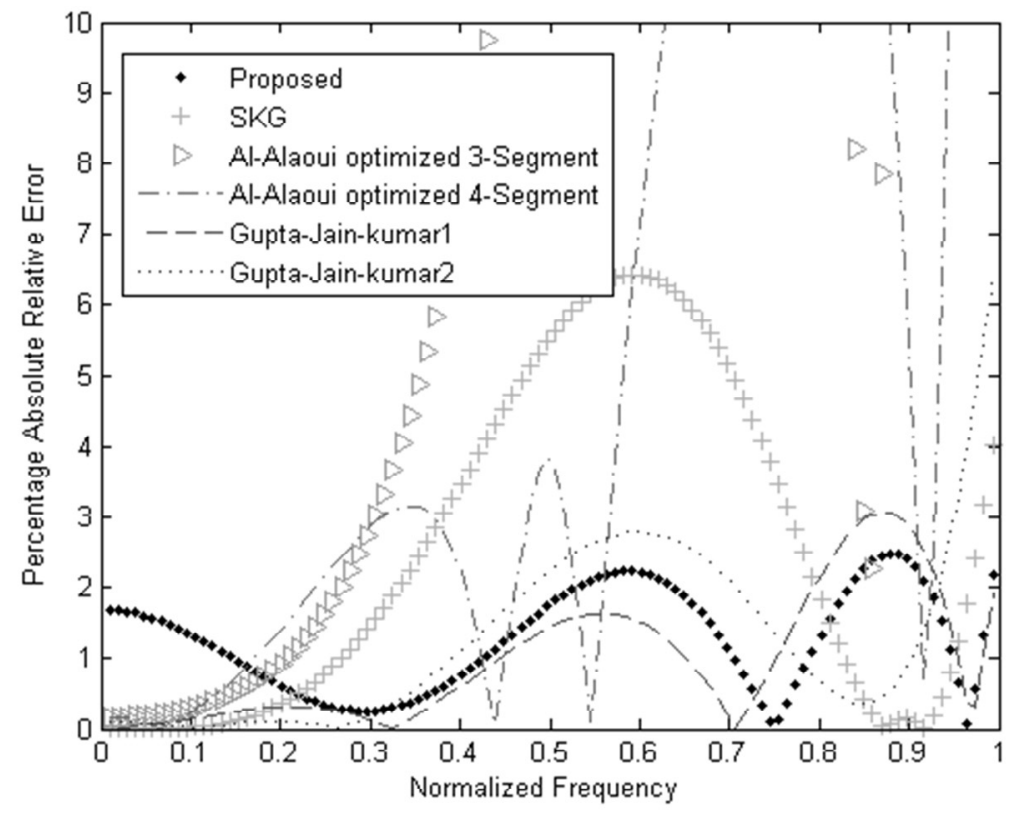

Fig. 3. PARE response of the proposed integrator $H_{N 5}(z), S K G$ integrator [2], Al-Alaoui optimized 3-Segment integrator [6], Al-Alaoui optimized 4-Segment integrator [6], Gupta-Jain-Kumar1 integrator [8], and Gupta-Jain-Kumar2 integrator [9]

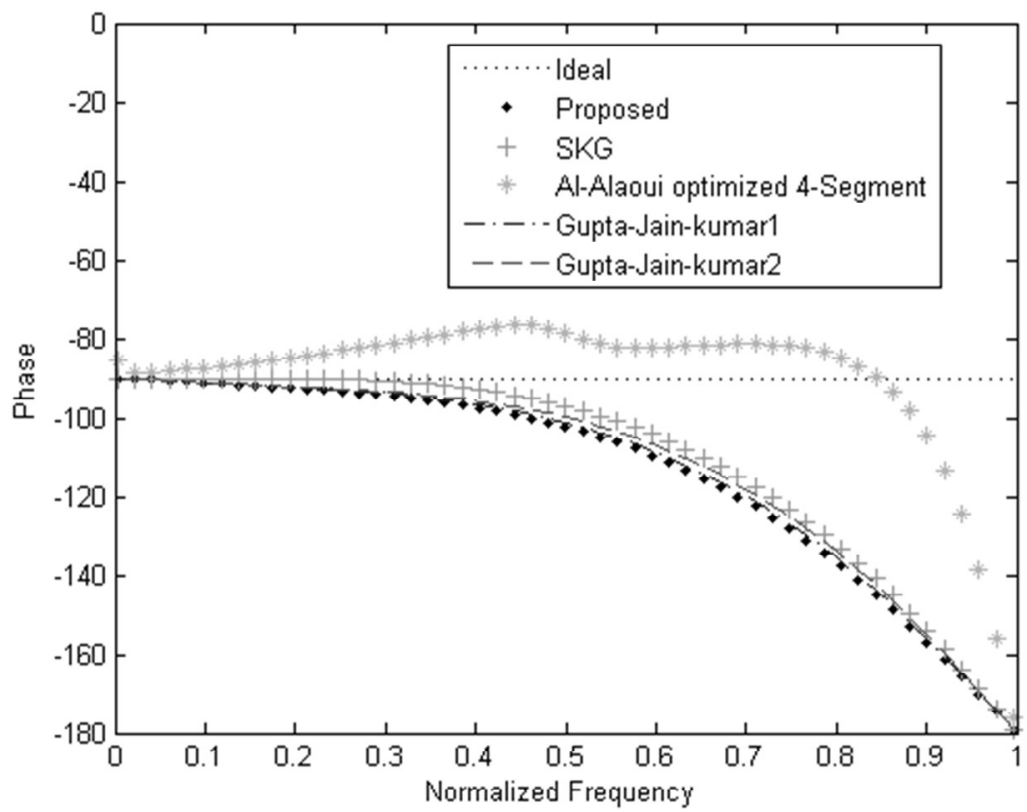

Fig. 4. Phase response of the proposed integrator HN5(z), SKG integrator [2], Al-Alaoui optimized 4-Segment integrator [6], Gupta-Jain-Kumar 1 integrator [8], and Gupta-Jain-Kumar 2 integrator [9] 
Table 1. Comparison between the existing and proposed integrators

\begin{tabular}{c|c|c}
\hline S. No. & Integrator & Maximum PARE over the Nyquist frequency range $0 \leq \omega \leq \pi$ radians \\
\hline 1. & Proposed & 2.4 \\
\hline 2. & Gupta-Jain-Kumar1[8] & 3.0 \\
\hline 3. & SKG [2] & 6.43 \\
\hline 4. & Gupta-Jain-Kumar2 [9] & 6.74 \\
\hline 5. & Al-Alaoui optimized 4-Segment [6] & 29.84 \\
\hline 6. & Al-Alaoui optimized 3-Segment [6] & 89.56 \\
\hline
\end{tabular}

\section{Design of the Proposed Digital Differentiator and its Comparison WITH THE EXISTING DIFFERENTIATORS}

\subsection{Design of the Proposed Differentiator}

New differentiators are obtained by inverting the transfer function of the designed integrators of $H_{N 1}(z), H_{N 2}(z), H_{N 3}(z), H_{N 4}(z)$ and $H_{N 5}(z)$ that are given in Eqs. $12,14,16,18$, and $20 \mathrm{~b}$ and then using the stabilization method described by Al-Alaoui [13]. By taking the inverse of these transfer functions, their poles, which lie outside the unit circle, are at $\mathrm{z}=-2.217, \mathrm{z}=-2.59, \mathrm{z}=-2.366, \mathrm{z}=-2.285$ and $\mathrm{z}=$ -2.4582 , respectively. These unstable poles are replaced by their inverse to get stable poles. Then, the denominators are multiplied by a factor of 2.217, 2.59, 2.366, 2.285, and 2.4582, respectively, to compensate for the change in amplitudes. After all of these operations, the resulting transfer functions of the designed recursive stable digital differentiators are obtained as:

$$
\begin{aligned}
& \mathrm{D}_{\mathrm{N} 1}(\mathrm{z})=\frac{\mathrm{z}^{2}(\mathrm{z}-1)}{0.8222 \mathrm{~T}(\mathrm{z}+0.4511)\left(\mathrm{z}^{2}-0.2455 \mathrm{z}+0.04407\right)} \\
& \mathrm{D}_{\mathrm{N} 2}(\mathrm{z})=\frac{\mathrm{z}^{2}(\mathrm{z}-1)}{0.8886 \mathrm{~T}(\mathrm{z}+0.386)\left(\mathrm{z}^{2}-0.2311 \mathrm{z}+0.0429\right)} \\
& \mathrm{D}_{\mathrm{N} 3}(\mathrm{z})=\frac{\mathrm{z}^{2}(\mathrm{z}-1)}{0.8848 \mathrm{~T}(\mathrm{z}+0.4226)\left(\mathrm{z}^{2}-0.2547 \mathrm{z}+0.04697\right)} \\
& \mathrm{D}_{\mathrm{N} 4}(\mathrm{z})=\frac{\mathrm{z}^{2}(\mathrm{z}-1)}{0.8711 \mathrm{~T}(\mathrm{z}+0.4376)\left(\mathrm{z}^{2}-0.247 \mathrm{z}+0.04543\right)} \\
& \mathrm{D}_{\mathrm{N} 5}(\mathrm{z})=\frac{\mathrm{z}^{2}(\mathrm{z}-1)}{0.8706 \mathrm{~T}(\mathrm{z}+0.4068)\left(\mathrm{z}^{2}-0.2190 \mathrm{z}+0.0497\right)}
\end{aligned}
$$

The PARE response of designed differentiators $D_{N 1}(z), D_{N 2}(z), D_{N 3}(z), D_{N 4}(z)$, and $D_{N 5}(z)$ are shown in Figure 5.

It is observed that the differentiator $D_{N 5}(z)$ has less PAREs, as compared to other designed 
differentiators over the entire Nyquist frequency range. Hence, it is specified as a proposed differentiator throughout this paper.

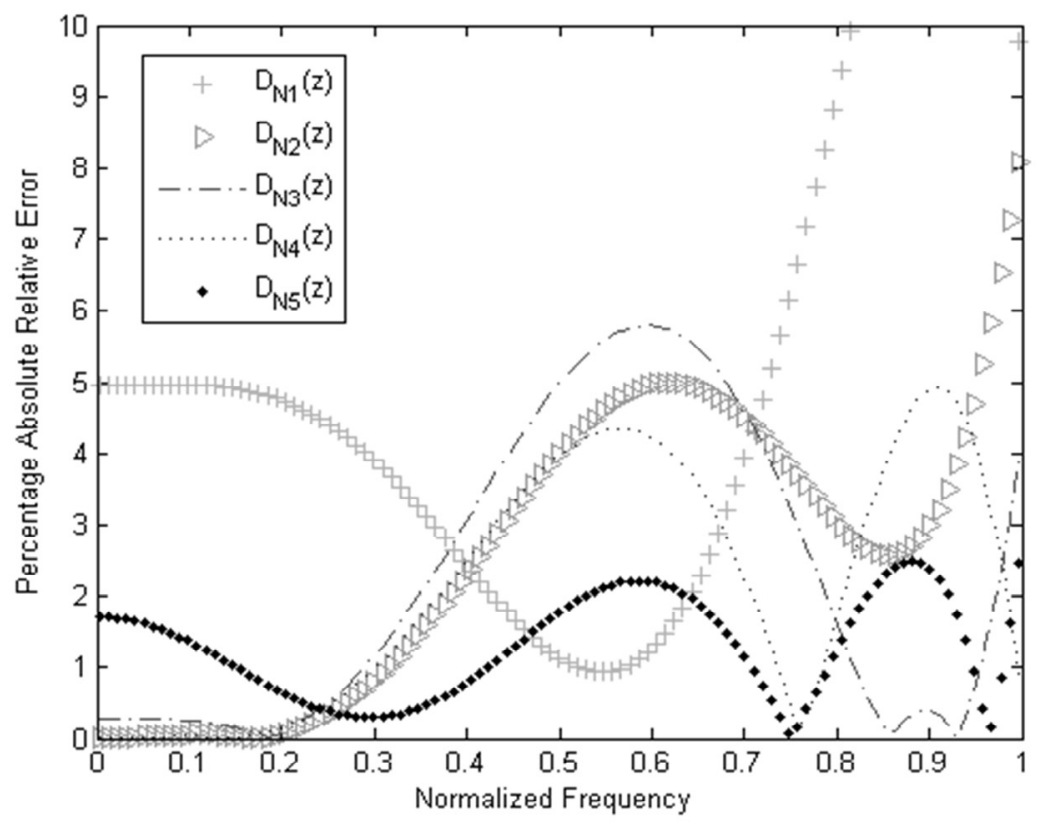

Fig. 5. PARE response of the designed differentiators $D_{N 1}(\mathrm{z}), D_{N 2}(\mathrm{z}), D_{N 3}(\mathrm{z}), D_{N 4}(\mathrm{z})$, and $D_{N 5}(\mathrm{z})$

\subsection{Comparison of the Proposed Differentiator with the Existing Differentiators}

It is appropriate to consider the existing differentiators that have been recently proposed for comparison. These are as follows:

Al-Alaoui optimized 3-Segment digital differentiator [6] is:

$$
\mathrm{D}_{\mathrm{AO} 3}(\mathrm{z})=\frac{\left(0.01903 \mathrm{z}^{3}-0.02905 \mathrm{z}^{2}+1.123 \mathrm{z}-1.181\right)}{\mathrm{T}\left(\mathrm{z}^{3}+0.1846 \mathrm{z}^{2}-0.001748 \mathrm{z}+0.03484\right)}
$$

Al-Alaoui optimized 4-Segment digital differentiator [6] is:

$$
D_{\mathrm{AO} 4}(\mathrm{z})=\frac{\left(238 \mathrm{z}^{4}+9.399 \mathrm{z}^{3}-3.322 \mathrm{z}^{2}+9.399 \mathrm{z}-241.3\right)}{\mathrm{T}\left(217 \mathrm{z}^{4}+259.9 \mathrm{z}^{3}+263.8 \mathrm{z}^{2}+259.9 \mathrm{z}+46.83\right)}
$$

Gupta-Jain-Kumar1 differentiator [8] is:

$$
\mathrm{D}_{\mathrm{GJK} 1}(\mathrm{z})=\frac{\mathrm{z}^{2}(\mathrm{z}-1)}{0.34 \mathrm{~T}(2.541)(\mathrm{z}+0.3935)\left(\mathrm{z}^{2}-0.2081 \mathrm{z}+0.03858\right)}
$$


Gupta-Jain-Kumar 2 differentiator [9] is:

$$
\mathrm{D}_{\mathrm{GJK} 2}(\mathrm{z})=\frac{\mathrm{z}^{2}(\mathrm{z}-1)}{0.329 \mathrm{~T}(2.663)(\mathrm{z}+0.3755)\left(\mathrm{z}^{2}-0.2079 \mathrm{z}+0.03864\right)}
$$

Pei-Hsu digital differentiator [14] is:

$$
\mathrm{D}_{\text {Pei-Hsu }}(\mathrm{z})=\left(\frac{3.0572}{-14.7667 \mathrm{~T}}\right)\left(\frac{0.1050 \mathrm{z}^{2}+0.4900 \mathrm{z}-0.5950}{-0.1050 \mathrm{z}^{2}-0.0332 \mathrm{z}+0.0027}\right)
$$

The frequency response (PARE and phase) of the proposed differentiator, along with existing ones, is shown in Figures 6-7 and their maximum PARE over the entire Nyquist frequency range is shown in Table 2.

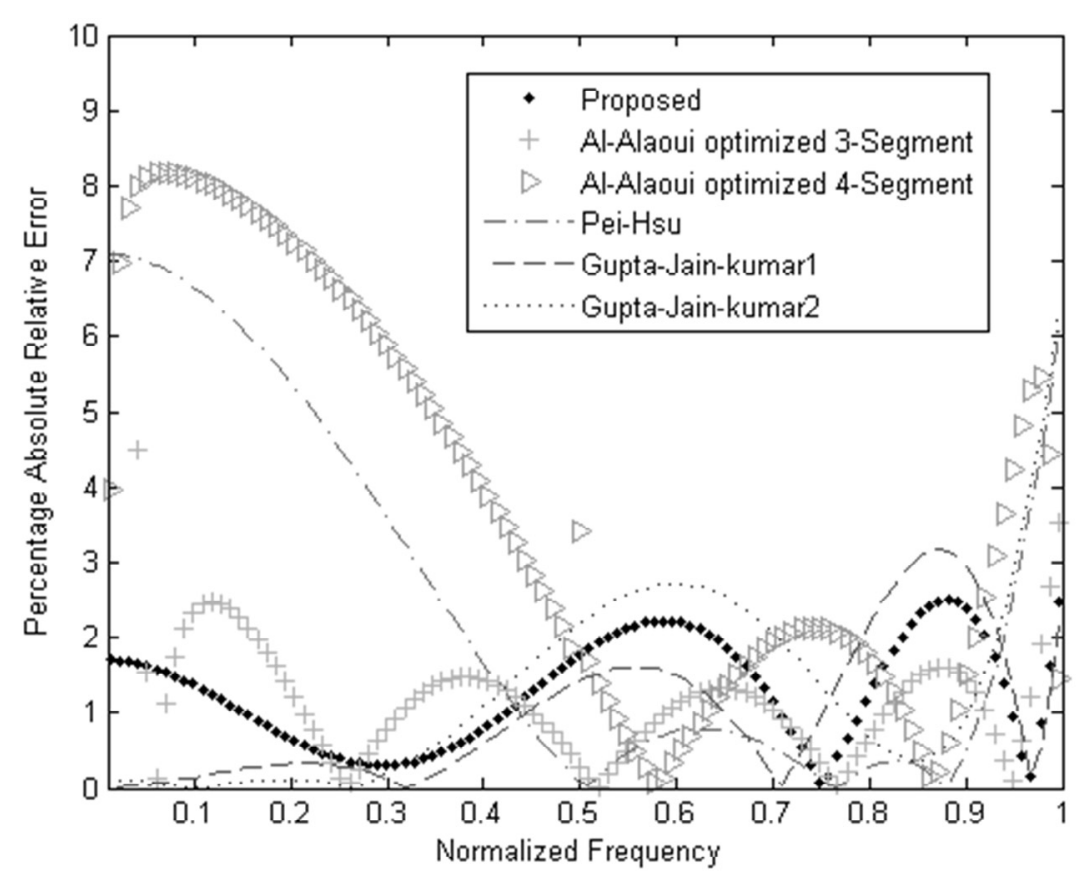

Fig. 6. PARE response of the proposed differentiator $D_{N 5}(\mathrm{z})$, Al-Alaoui optimized 3-Segment differentiator [6], Al-Alaoui optimized 4-Segment differentiator [6], Gupta-Jain-Kumar 1 differentiator [8], Gupta-Jain-Kumar 2 differentiator [9], and Pei-Hsu differentiator [14] 


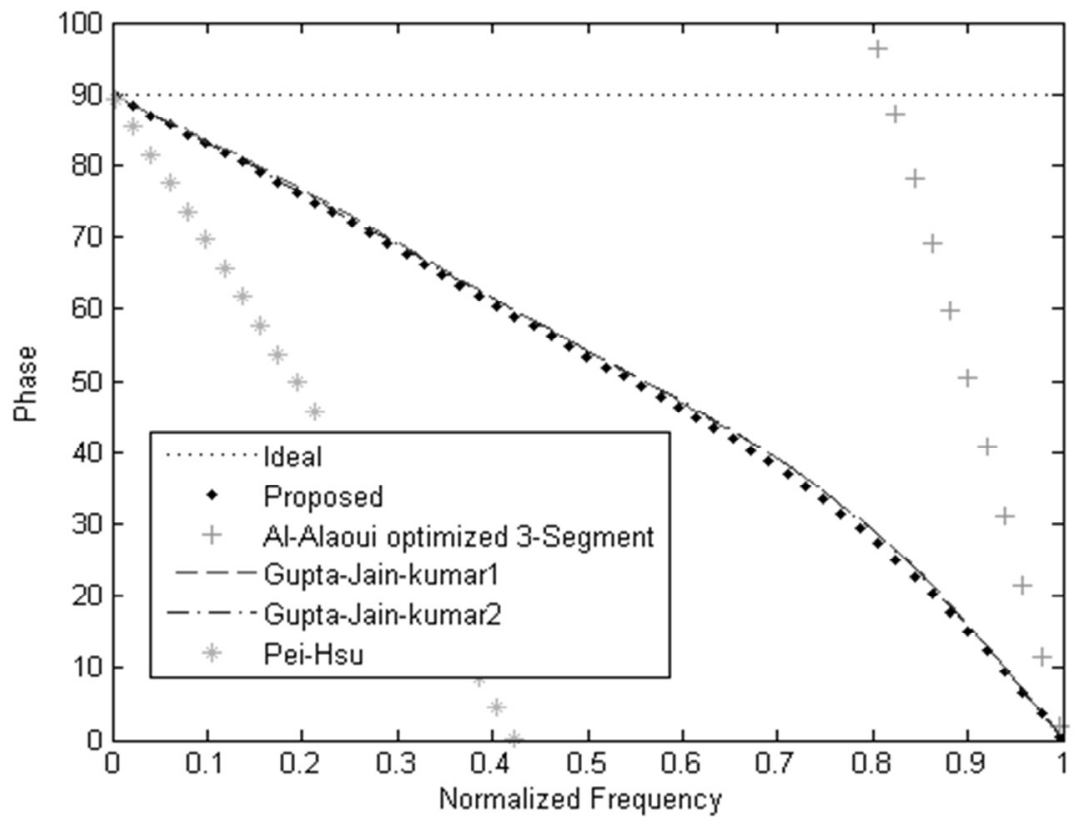

Fig. 7. Phase response of the proposed differentiator $D_{N 5}(z)$, Al-Alaoui optimized 3-Segment differentiator [6], Gupta-Jain-Kumar 1 differentiator [8], Gupta-Jain-Kumar 2 differentiator [9], and Pei-Hsu differentiator [14]

Table 2. Comparison between the existing and proposed differentiators

\begin{tabular}{c|c|c}
\hline S. No. & Differentiator & $\begin{array}{c}\text { Maximum PARE over the Nyquist frequency range } \\
\boldsymbol{0} \leq \boldsymbol{\omega} \leq \boldsymbol{\pi} \text { radians }\end{array}$ \\
\hline 1. & Proposed & 2.4 \\
\hline 2. & Gupta-Jain-Kumar1[8] & 3.0 \\
\hline 3. & Gupta-Jain-Kumar2 [9] & 6.31 \\
\hline 4. & Pei-Hsu [14] & 7.09 \\
\hline 5. & Al-Alaoui optimized 4-Segment [6] & 47.95 \\
\hline 6. & Al-Alaoui optimized 3-Segment [6] & 466.63 \\
\hline
\end{tabular}

Figure 6 and Table 2 assert that the proposed differentiator $D_{\mathrm{N} 5}(\mathrm{z})$ is the closest approximation of the ideal differentiator as compared to the existing ones.It can be seen from Figure 7 that the maximum phase deviation of the proposed differentiator from the ideal linear phase response is $10.1^{\circ}$ (which occurs at $\omega=0.74 \pi$ radians). While the maximum phase deviation of the existing differentiators Al-Alaoui optimized 3-Segment [6], Gupta-JainKumar 1 [8], Gupta-Jain-Kumar 2 [9], and Pei and Hsu [14] from the ideal linear phase response is $48.3^{\circ}$ (which occurs at $\omega=0.098 \pi$ radians), $12.0^{\circ}$ (which occurs at $\omega=0.75 \pi$ radians), $11.0^{\circ}$ (which occurs at $\omega=0.71 \pi$ radians), and $29.0^{\circ}$ (which occurs at $\omega=0.61 \pi$ radians), respectively. It can be noticed (from Figures6 and 7 and Table 1) that the proposed differentiator $D_{N 5}(z)$ outperforms all the existing differentiatorsin magnitude and phase response over the entire Nyquist frequency range. 


\section{Application of the Proposed Digital Differentiator in Image PROCESSING}

Digital integrators and differentiators are important components in almost all engineering disciplines, including in control, communication, biomedical, and image processing applications. Edge detection is used to remove useless information and retains the important information in an image. It is a process that is used to identify and locate sharp discontinuities in an image. The discontinuities are abrupt changes in pixel intensity, which characterize the boundaries of objects in a scene. There are many ways to perform edge detection. Classical methods of edge detection [10-12] involve convolving the image with an operator, which is constructed to be sensitive to large gradients in the image while returning values of zero in uniform regions. Some of the edge detection operators that are discussed in this work are Roberts, Sobel, Prewitt, Laplacian of Gaussian, Zerocross, and Canny [10-12].

We performed edge detection using our proposed differentiator (designed in Section 3.1). The algorithm is defined as follows: in the first step, if an image is imported as grayscale then take it as it is; otherwise first convert it to grayscale. In the second step, the direction of the edge is computed using the gradient in the $x$ and $y$ directions. Here, gradients are estimated using the proposed differentiator $\mathrm{D}_{\mathrm{N} 5}(\mathrm{z})$.

$$
\mathrm{D}_{\mathrm{N} 5}(\mathrm{z})=\frac{\left(\mathrm{z}^{3}-\mathrm{z}^{2}\right)}{\left(0.8706 \mathrm{z}^{3}+0.1635 \mathrm{z}^{2}-0.0342 \mathrm{z}+0.0176\right)}
$$

The time domain difference equation can be written as:

$$
y(n)=\left(\frac{1}{0.0176}\right)\{x(n-3)-x(n-2)+0.0342 y(n-1)-0.1635 y(n-2)-0.8706 y(n-3)\}
$$

Consider that an image $\mathrm{f}(\mathrm{x}, \mathrm{y})$ and the gradient of the image can be considered as:

$$
\nabla \mathrm{f}(\mathrm{x}, \mathrm{y})=\frac{\delta f(x, y)}{\delta x} u_{x}+\frac{\delta f(x, y)}{\delta y} u_{y}=G_{x} u_{x}+G_{y} u_{y}
$$

Where, $\mathrm{u}_{\mathrm{x}}$ and $\mathrm{u}_{\mathrm{y}}$ are the unit vectors in $\mathrm{x}$ and $\mathrm{y}$ directions. The approximated magnitude of the gradient is:

$$
|G|=\sqrt{G_{x}^{2}+G_{y}^{2}}
$$

Considering that $\mathrm{x}[\mathrm{n}]=\mathrm{f}(\mathrm{x}, \mathrm{y})$ and applying it to a digital differentiator the outputs are calculated both in horizontal $(x)$ and vertical $(y)$ directions individually, and the gradient is calculated using Eq. (34).

In the third step, weak edges are eliminated. Then, in the last step, the threshold is applied to finalize the edges. The proposed edge detection algorithm wassuccessfully implemented on the software MATLAB 7 and applied tothree different kinds of images to confirm its robustness.

Following are the four different criteria which are used to evaluate the performance of an edge detection algorithm: first, the probability of marking something as an edge which is actually not 
an edge. Second is the probability of failing to mark an edge which is actually an edge. Third is the error in calculation of the edge angle and the fourth is the mean square distance of the edge approximated from the true edge. However, in order to check the third and fourth criteria, an exact map of the edges in an image must be known, and generally this is not available. Here, for evaluation, only the first two criteria are considered.

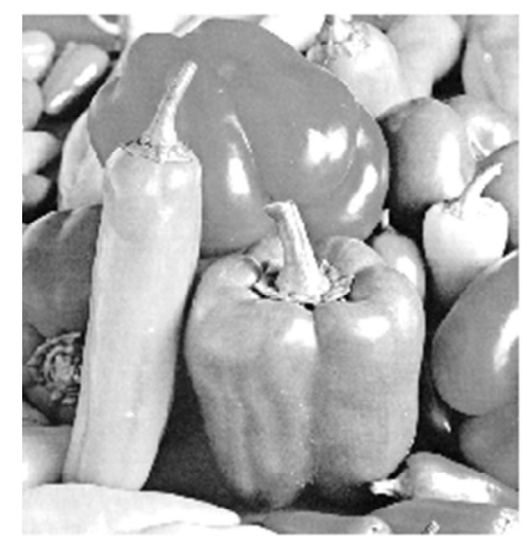

(a) Original image

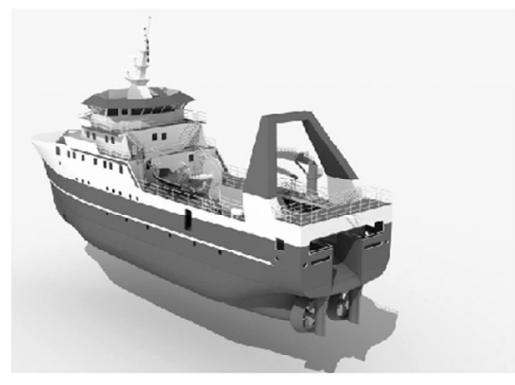

(c) Original image

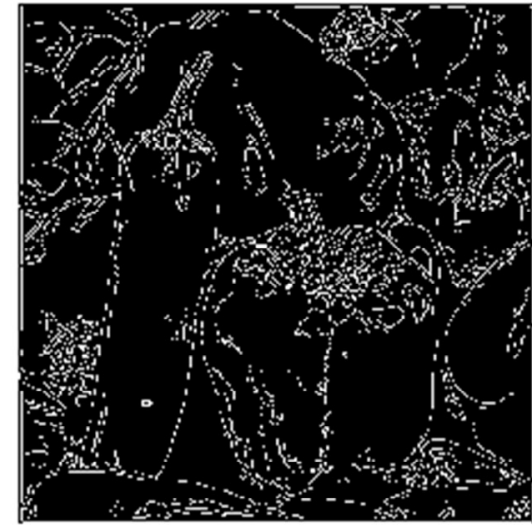

(b) Edge image using the proposed differentiator

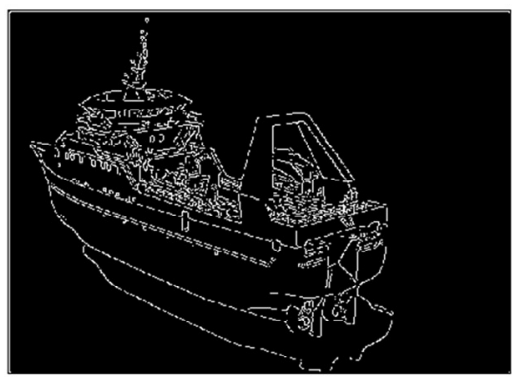

(d) Edge image using the proposed differentiator

Fig. 8. Original images and test results: original images; (a), (c), results of edge detection using proposed differentiator; (b), (d)

It is demonstrated in Figure 8 that the proposed differentiator successfully performs edge detection.To show the effectiveness of the proposed differentiator in edge detection, its results were compared with other methods on the popular Lena image and they are shown in Figures 9 and 10 . 

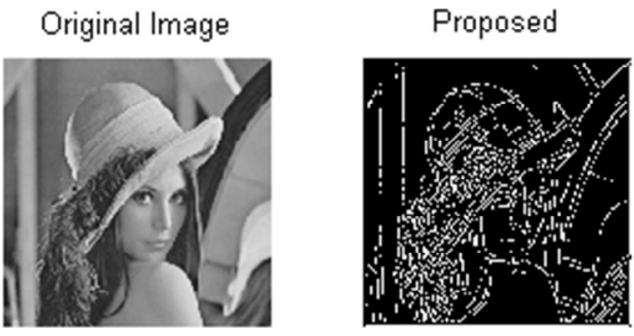

Al-Alaoui optimized 3-Segment

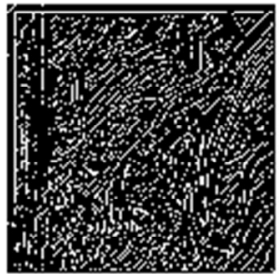

Pei and Hsu

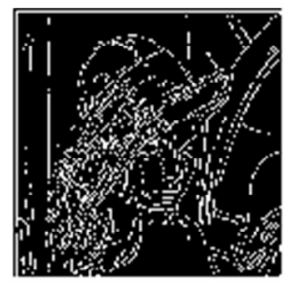

G.JK1

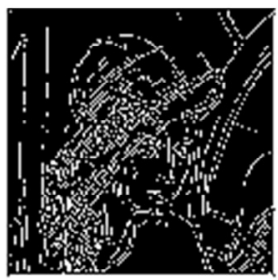

GJK2

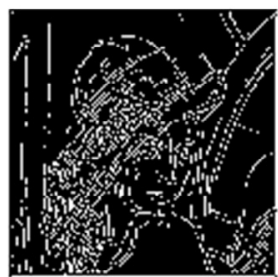

Fig. 9. Results of edge detection using the proposed differentiator $D_{N 5}(z)$ and the existing differentiators of: Al-Alaoui optimized 3-Segment [6], Gupta-Jain-Kumar1 (GJK1) [8], Gupta-Jain-Kumar 2 (GJK2) [9], and Pei and Hsu [14]

Prewitt

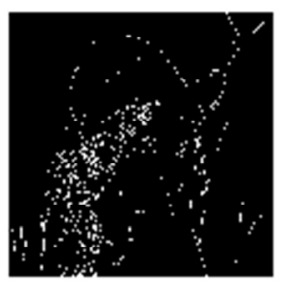

Canny

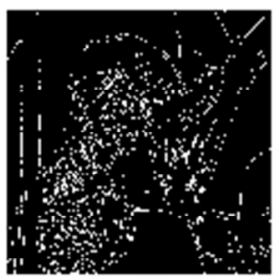

Sobel

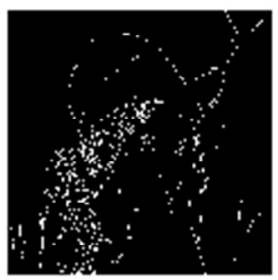

\section{LOG}

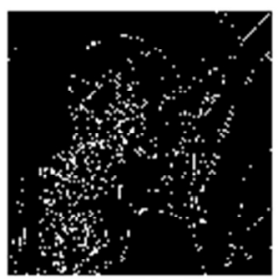

\section{Roberts}

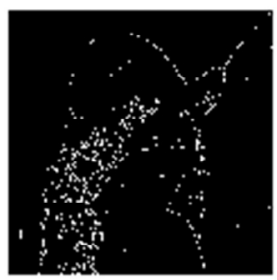

Zerocross

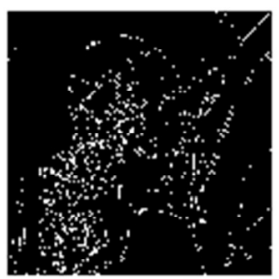

Fig. 10. Results of edge detection using the classical operators of Prewitt, Sobel, Roberts, Canny, LOG, and Zerocross [10-12] 
Figures 9 and 10 show that the proposed differentiator has superior performance in edge detection, as compared to other differentiators.

\section{Conclusions}

In this paper, new differintegrators were designed. The absolute relative error percentage of the proposed integrator and differentiator is $\leq 2.4$ over the entire Nyquist frequency range: $0 \leq$ $\omega \leq \pi$ radians. It has been shown that the proposed differintegrator outperforms the existing ones in terms of both magnitude and phase response. The practical ability of the proposed differentiator is demonstrated by applying it for edge detection in an image processing application. The proposed differentiator is applied for three different kinds of images and the test results were compared with existing differentiators and operators. The comparisons made in this paper were carried out through visual observations using only the software MATLAB 7. From the results obtained, it can be concluded that the proposed differentiator proves to be very effective with less false edges. It gives superior performance than the operators Sobel, Prewitt, Roberts, LOG, Zerocross, and Canny and than other existing differentiators.

\section{REFERENCES}

[1] M. A. Al-Alaoui, "Novel digital integrator and differentiator," Electronics Letters, vol. 29, no. 4, pp. 376-378, 1993.

[2] A. M. Schneider, J. T. Kaneshige and F. Groutage, "Higher order s-to-z mapping functions and their application in digitizing continuous-time filters," Proceedings of the IEEE, vol. 79, no. 11, pp. 1661$1674,1991$.

[3] N. Papamarkos and C. Chamzas, "A new approach for the design of digital integrators," IEEE Transactions on Circuits and Systems I: Fundamental Theory and Applications, vol. 43, no. 9, pp. 785-791, 1996.

[4] N. Q. Ngo, "A new approach for the design of wideband digital integrator and differentiator," IEEE Transactions on Circuits and Systems II: Express Briefs, vol. 53, no. 9, pp. 936-940, 2006.

[5] M. A. Al-Alaoui, "Using fractional delay to control the magnitudes and phases of integrators and differentiators," IET Signal Processing, vol. 1, no. 2, pp. 107-119, 2007.

[6] M. A. Al-Alaoui, "Class of digital integrators and differentiators," IET Signal Processing, vol. 5, no. 2, pp. 251-260, 2011.

[7] M. Gupta, M. Jain, and B. Kumar, "Wideband digital integrator," in Proceeding of the IEEE International Conference on Multimedia, Signal Processing and Communication Technologies, Aligarh, India, 2009, pp. 106-108.

[8] M. Gupta, M. Jain, and B. Kumar, "Novel class of stable wideband recursive digital integrators and differentiators," IET Signal Processing, vol. 4, no. 5, pp. 560-566, 2010.

[9] M. Gupta, M. Jain, and B. Kumar, "Recursive wideband digital integrator and differentiator," International Journal of Circuit Theory and Applications, vol. 39, no. 7, pp. 775-782, 2011.

[10] D. Marr and E. Hildreth, "Theory of edge detection," Proceedings of the Royal Society of London. Series B. Biological Sciences, vol. 207, no. 1167, pp. 187-217, 1980.

[11] J. Canny, "A computational approach to edge detection," IEEE Transactions on Pattern Analysis and Machine Intelligence, vol. PAMI-8, no. 6, pp. 679-698, 1986.

[12] D. Demigny, "On optimal linear filtering for edge detection," IEEE Transactions on Image Processing, vol. 11, no. 7, pp. 728-737, 2002.

[13] M. A. Al-Alaoui, "Novel approach to designing digital differentiators," Electronics Letters, vol. 28, no. 15, pp. 1376-1378, 1992.

[14] S. C. Pei and H. J. Hsu, "Fractional bilinear transform for analog-to-digital conversion," IEEE Transactions on Signal Processing, vol. 56, no. 5, pp. 2122-2127, 2008. 


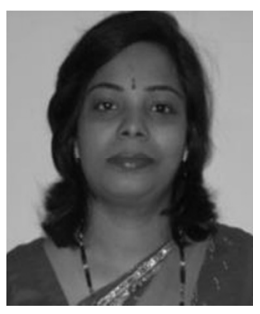

\section{Madhu Jain}

She received B.E. degree in Electronics and Communication Engineering from University of Rajasthan in 2003 and M. Tech. in Signal Processing from Netaji Subhas Institute of Technology, New Delhi in 2009. She is pursuing Ph. D. in IIT Delhi and currently working as Assistant Professor in Electronics \& Communication Engineering Department of Jaypee Institute of Information Technology, Noida. Her teaching and research interests are Digital Signal Processing, Signal Systemsand Embedded System. She has co-authored 10 research papers in the above areas in various international journals and conferences.

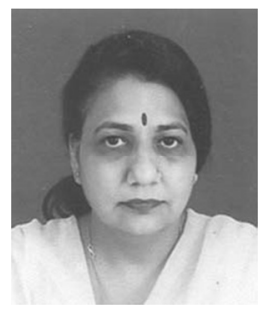

\section{Maneesha Gupta}

She received B.E. and M.E. in Electronics \& Communication Engineering from Government Engineering College, Jabalpur in 1981 and 1983 respectively and Ph.D. in Electronics Engineering from Indian Institute of Technology, Delhi in 1990. She is currently working as Professor in Electronics \& Communication Engineering Department of Netaji Subhas Institute of Technology, New Delhi since 2000. Her teaching and research interests are Switched Capacitors Circuits and Analog Signal processing. She has co-authored over 40 research papers in the above areas in various international/ national journals and conferences.

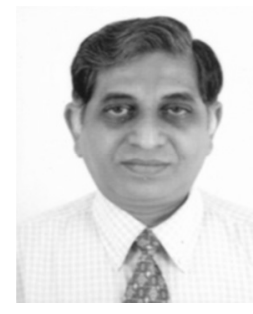

\section{N.K. Jain}

He received B.E. from Jabalpur University in 1973, M. Tech. and Ph.D from IIT Delhi in 1975 and 1985, respectively. He is currently working as Chief Design Engineer (SG) in IIT Delhi. He is also serving as Head, IDD Centre since May 2009. He has co-authored 10 research papers in various conferences/ proceedings. He has developed 5 technologies and has completed 26 research projects. He has also contributed 11 chapters in 6 books. 